\title{
Advances in X-ray Micro-Spectroscopy of Heterogeneous Catalysts
}

\author{
$\underline{\text { Bert M. Weckhuysen }}^{1, *}$
}

1. Inorganic Chemistry and Catalysis group, Debye Institute for Nanomaterials Science, Utrecht University, Utrecht, the Netherlands.

* Corresponding author, b.m.weckhuysen@uu.nl

Catalysis is a very important technology in modern society as evidenced by the fact that more than $85 \%$ of all fuels and chemicals are made via one or more catalytic reactions. To design very active, selective and stable solid catalysts it is necessary to understand how catalysts exactly operate and why they deactivate when a chemical reaction progresses. Experimental tools for the analysis of solid catalysts include both microscopy and spectroscopy, which preferentially cover multiple length scales - from the atomic length scale when looking at the active sites to tens of metres when studying reactors. Analysis is also required over multiple time scales ranging from the time a reactant needs to diffuse to the active site and subsequently adsorbs and reacts to the time during which a catalyst deactivates. Finally, to reliably assess how a catalyst works it is desirable to study it under real catalytic conditions. This poses requirements to the applied technique that can be limiting, because high temperatures and pressures are often needed. It should also be clear from the above that there is not a single technique that can satisfy all the needs of solid catalyst characterization, which motivates the use of a multi-technique approaches.

Amongst the characterization methods available, X-ray based techniques have established themselves as inevitable for solid catalyst characterization. The last years have been dominated by two main developments: first, the development of in-situ or operando spectroscopy, and second, the development of high spatial and temporal resolution mapping of catalyst composition, structure and morphology. In this plenary lecture, I will present an overview of these instrumental developments in which the progress made in the broad field of X-ray micro-spectroscopy (XRM) for investivating heterogeneous catalysts is discussed. This will be done by using two showcase examples, namely the Fischer-Tropsch Synthesis (FTS) catalyst as an archetypical example for the use of operando XRM, and the Fluid Catalytic Cracking (FCC) catalyst as an example to illustrate the application of XRM for high spatial resolution mapping of chemical composition as well as structure and porosity. In what follows, I will highlight of these developments and also offer some historical perspectives to put these studies in a broader context.

Most probably the earliest studies using synchrotron radiation-based XRM, investigated three types of heterogeneous catalysts, namely polyethylene polymerization particles hydrotreating catalysts, and FCC particles [1,2]. For studying FCC particles, Computed Tomography (CT) was combined with X-ray Fluorescence (XRF) tomography with a beam size of $10 \mu \mathrm{m}$ produced by a mechanical collimator. By using this set-up, the distribution of the metal poisons $\mathrm{Fe}, \mathrm{Ni}$ and $\mathrm{V}$, regularly present in (partially) deactivated FCC catalyst particles, could be visualized in virtual cross-sections obtained by tomography. These 2-D XRF maps were complemented by micrometre resolution CT measurements providing a higher resolution ( $3 \mu \mathrm{m} \times 3 \mu \mathrm{m}$ pixel size) virtual slice through an FCC particle. The authors made it possible to reveal the inhomogeneous distribution of regions of high and low contrast were interpreted as the zeolite phases embedded in the matrix of an FCC catalyst particle consisting of clay, $\mathrm{SiO}_{2}$, and $\mathrm{Al}_{2} \mathrm{O}_{3}$. In comparison with the 2-D XRF data, it was deduced that the high contrast surface regions must be regions enriched with the contaminating metals. Although this interpretation highly simplified the true FCC 
catalyst structure, which not only contains matrix elements (i.e., $\mathrm{SiO}_{2}, \mathrm{Al}_{2} \mathrm{O}_{3}$ and clay) and zeolites, but also micrometer-sized pores, this study showed the potential of XRM for studying single catalyst particles.

It was these initial capabilities of XRM, which has inspired our group to embark on the use of both Scanning Transmission X-ray Microscopy (STXM) and Transmission X-ray Microscopy (TXM) to investigate a wide variety of single catalyst particles, including FCC catalysts, FTS catalysts, methanolto-hydrocarbon (MTH) catalysts and Phillips ethylene polymerization catalysts [3]. STXM makes use of soft X-rays, while TXM makes use of hard X-rays; thereby providing complementary information on the catalyst system under study. For both XRM methods we have developed over the years in-situ and operando spectroscopy set-ups, most often in long-term cooperation programs with beamline scientists, to allow to capture 2-D and 3-D snapshots of e.g. a Fe- or Co-based FTS catalysts under CO hydrogenation conditions at elevated temperatures and pressures [4-7]. It should be clear that such experiments are very challenging, but also provides a wealth of information on the dynamics of a single catalyst particle under realistic reaction condition. For example, we have been able to capture the active state of a $\mathrm{Co} / \mathrm{TiO}_{2} \mathrm{FTS}$ particle, and also determine the spatiotemporal dynamics of the organics formed within this particle.

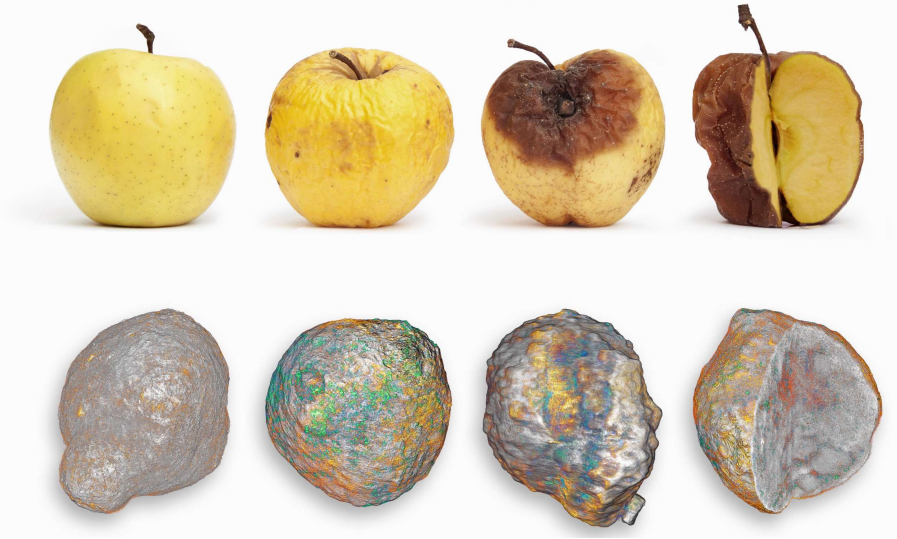

Figure. 1. (top row) Pictorial to illustrate the life and death of a single Fluid Catalytic Cracking (FCC) particle by making the comparison with the rotting process of an apple; and (bottom row) 3-D TXM images of an FCC catalyst particle. From left to right, from a fresh catalyst particle to a catalyst particle rich in metal poisons, such as iron (orange-red) and nickel (green-blue). Reproduced from reference [10].

Another system, which we have been researching in great detail, both with TXM and STXM, in combination with e.g. 3-D XRF, is the FCC catalyst [8-12]. In 2015, we have published our first studies, in which 3-D TXM was performed on single FCC catalyst particles, which have been taken from an oil refinery [8-10]. By doing so, and comparing the chemical composition as well as internal pore structure and connectivity for different catalyst particles it was possible to probe the life and death of an FCC particle; and determine the causes for its deactivation. More specifically, we have been able to identify what the role of $\mathrm{Fe}$ and $\mathrm{Ni}$ are on the pore accessibility, as outlined in Figure 1. Figure 1 makes the catalyst ageing with the rotting process of an apple. It was found that $\mathrm{Fe}$ and Ni changes the the porosity, especially at the outer rim of the catalyst particles, thereby limiting their accessibility towards reactants; hence the catalyst particles will gradually becomes less accessible, thereby losing their catalytic performance. These initial TXM studies have been complemented with a detailed study in which 3-D XRF was combined with TXM [11]; as well as by a STXM study [12]. The latter method turned out to be very instrumental as it was possible to determine the location of the zeolite domains with an FCC catalyst particle, and by using a set of reference spectra for octahedral and tetrahedral $\mathrm{Al}$ it was possible to determine the degree of zeolite dealumination as a function of the content of metal poisons in the catalyst particle, as well as to 
determine the spatial distribution of octahedral Al within a single catalyst particle.

From the above discussions it should become apparent that important developments in the field of solid catalyst characterization is taking place. Improving the temporal and spatial has been the ultimate goal of scientists. However, we have not yet realized the dream of recording the ultimate 'molecular movie' of a solid catalyst under true operando conditions, as pictorially illustrated in Figure 2. To realize this dream, it is important to follow in real time both the inorganic and organic part of a catalytic event, which requires most probably a combination of tools. Finally, to understand and link the micro- and macro-kinetics that determine catalyst performance, this tool (set) should furthermore have a spectroscopic lens that is capable of capturing all length scales involved. Tackling these challenges will pave the way for discoveries in the quest of spatial and temporal explorations of solid catalysts [13].
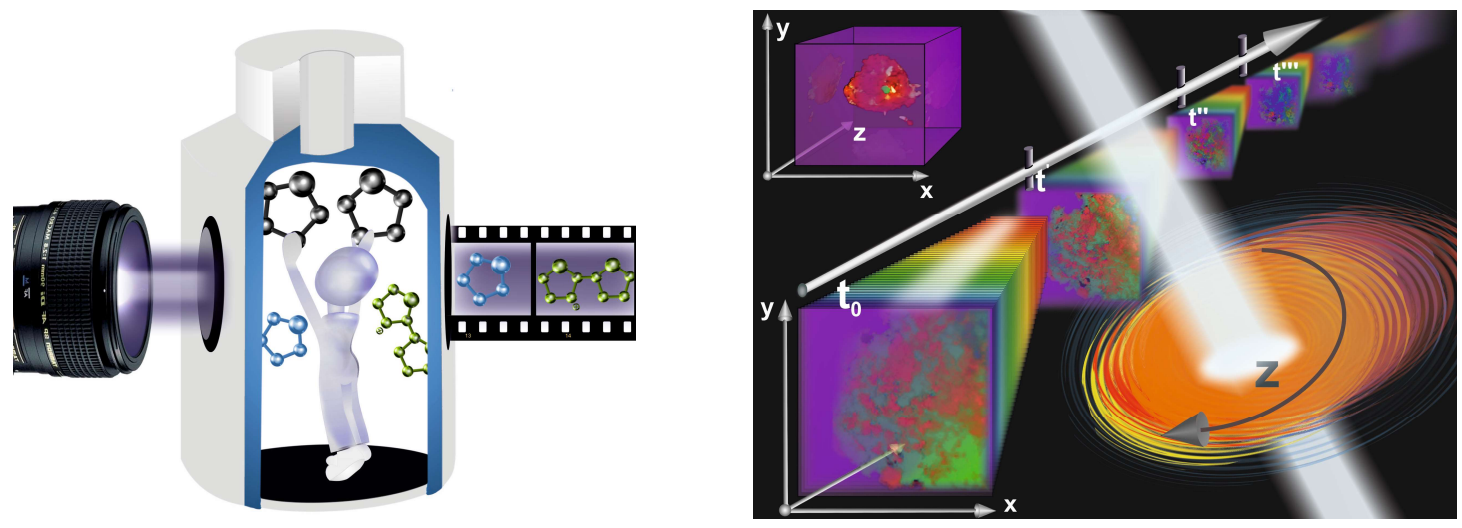

Figure. 2. (left) Pictorial to illustrate the dream to make a molecular movie of a solid catalyst under realistic reaction conditions allowing to shed insight in how a catalyst operates and deactivates; and (right) Spatial and temporal mapping of a catalyst particle in 3-D at the sub-nanometer scale, and at the same time monitoring the chemistry taking placewithin this particle. Increasing X-ray brilliance and advancement in detector technology as well as data mining tools will allow us to make this dream reality.

References:

[1] W.C. Conner et al, Macromolecules 23 (1990) 4742-4747

[2] K.W. Jones et al, Nucl. Instrum. Meth. B 56-57 (1991) 427-432

[3] I.L.C. Buurmans et al, Nat. Chem. 4 (2012) 873-886

[4] E. de Smit et al, Nature 456 (2008) 222-239

[5] I. Gonzalez-Jimenez et al, Angew. Chem. Int. Ed. 51 (2012) 11986-11990

[6] K.H. Cats et al, Chem. Commun. 49 (2013) 4622-4624

[7] K.H. Cats et al, Catal. Sci. Technol. 6 (2016) 4438-4449

[8] F. Meirer et al, J. Am. Chem. Soc. 137 (2015) 102-105

[9] F. Meirer et al, Chem. Commun. 51 (2015) 8097-8100

[10] F. Meirer et al, Sci. Adv. 1 (2015) e1400199

[11] Y. Liu et al, Nat. Commun. 7 (2016) 12634

[12] S. Kalirai et al, Angew. Chem. Int. Ed. 55 (2016) 11134-11138

[13] The author acknowledges funding from the European Research Council (ERC) Advanced Grant (No. 321140), a MCEC Gravitation Program of the Netherlands Organization for Scientific Research (NWO). 
https://doi.org/10.1017/S1431927618014344 Published online by Cambridge University Press 